\title{
8 Präsenz und Flow - im Blick des Fließenden Gewahrseins
}

\author{
Gabriele Plesse-St. Clair und Frithjof Paulig
}

\section{Hinführung}

„Das größte Geschenk, das wir uns als Menschen einander machen können, ist die ungeteilte Aufmerksamkeit." (frei nach Carl Rogers)

Kennen wir nicht alle die Momente, wo das wirkliche Dasein eines Gegenübers unsere Herzen ergreift und wir unser Menschsein spüren und das nicht nur in der Beziehung zueinander, sondern auch in der Beziehung zu uns selbst, zu unserer Welt und dem, was wir das „Größere“ nennen können, den Raum oder das Tao, dass alles einbezieht?

Vielleicht trifft uns der offene Blick eines Kindes, wir versinken als Liebende im Augenblick, wir hören die Stille in der Lücke zwischen 2 Gedanken, wir spüren den Raum, der sich auftut, wenn wir Innehalten, wir erfahren die Qualität eines Lebendigseins, wenn wir ganz in dem aufgehen, was wir tun - und „der Tänzer zum Tanz wird“".

Im Folgenden gehen wir auf das Verständnis von Präsenz und Flow ein und zeigen dann, wie das Fließende Gewahrsein (FlieG), ein Schlüssel aus dem Spektrum orgodynamischer Bewusstseinsarbeit, in der Praxis der Bewusstseinsschulung angewendet werden kann.

\section{Präsenz}

Recherchiert man im Internet wird deutlich, wie sehr der materielle, messbare, sichtbare Aspekt von Gegenwärtigkeit in der Verkopplung von Werbung und Waren betont 
wird. Präsenz wird reduziert zu einer Anwesenheits- und Vorhandenseinsfunktion. Präsenzfähigkeit wird im alltäglichen Sprachgebrauch häufig auch mit einer rein mentalen Bewusstseinskompetenz gleichgesetzt.

Als Bewusstseinsmodus ist Präsenz jedoch vielmehr. Es geht um die menschliche Fähigkeit, die Gegenwart bewusst wahrnehmen zu können und damit am Jetztmoment zu partizipieren.

Präsenz enthält verschiedene Zugänge und Qualitäten, z.B. Achtsamkeit, ungeteilte Aufmerksamkeit, Gewahrsein, Anfängergeist. Ihre Gemeinsamkeit liegt darin, dass sie den Augenblick, den Moment, das Jetzt unmittelbar berühren und erfassen.

- Achtsamkeit beinhaltet z.B. Achtung im Sinne von Wertschätzung, aber auch Achtung als Qualität des Achtgebens, der Vorsicht oder der Warnung, Acht zu geben. Hier zeigen sich verschiedenste Perspektiven, sich auf den Moment zu beziehen, ihn offen zu begrüßen oder eingrenzend auszuwählen.

- Aufmerksamkeit ist oft ein Synonym für Konzentration, damit zielgerichtet und schnell an einen bestimmten Inhalt gebunden. Ungeteilte Aufmerksamkeit weist auf die Fähigkeit hin, sich fokussiert und unabgelenkt einem Objekt, einem Menschen, sich selbst etc. zuwenden zu können.

- Der aus der Zen-Tradition stammende Begriff „Anfängergeist“" weist auf eine Qualität der Neugierde und Frische, mit der das Leben wahrgenommen werden kann.

Dem Begriff Gewahr-Sein, Gewahr-Werden könnte eine mehr prozessuale, fließende Qualität zugeordnet werden. Jeder Prozess des Gegenwärtigsein braucht auch die Bereitschaft, das soeben wahrgenommene wieder loszulassen, um offen zu bleiben für den Moment, für das Jetzt in seiner ganzen Fülle. Nur das Jetzt ist der „Ort“ für Veränderung, Transformation, Wachstum und Lernen.

Auch in der Neurobiologie wird das Jetzt als „einzig mögliches Einflussfenster zur Veränderung“ betont, wobei es um das Zusammenspiel der 3 Qualitäten Flexibilität, Halten und Fokussieren geht (Gottwald 2006), die in ihrem Zusammenspiel zu einem fließenden Prozess des Gegenwärtigseins werden.

Belschners Verständnis von Präsenz unterscheidet zwischen Alltags-Wach-Bewusstsein, empathischer und nondualer Präsenz, wobei die Modulation dieser verschiedenen Modi im Mittelpunkt bleibt, welche in die Bewusstseinsweite führen (Belschner 2006).

Goldstein und Kornfield betonen in ihrem Verständnis das Zusammenspiel von Geist und Herz (Goldstein, Kornfield 1993), so wie ja auch in vielen spirituellen Traditionen vom emphatischen und dem geistigen Weg der Achtsamkeit als universellem Medium, mit dem „Größeren“, dem Göttlichen in Verbindung zu treten, gesprochen wird.

Wenig wird jedoch von der Bedeutung der Körperpräsenz als einem Medium, den Jetztmoment zu erfassen gesprochen, ja gerade im Blick der spirituellen Ausrichtung wird dieser häufig ausgeklammert.

Im orgodynamischen Verständnis wird die Körperpräsenz genauso beachtet, wie kognitive Präsenzfähigkeiten. Die offenen Sinne und ein waches Spürbewusstsein im Körper bereiten den Boden für eine Gegenwärtigkeit, die eben nicht nur kognitiv 
oder fühlend präsent ist, sondern auch die körperintuitive Wachheit für den Augenblick mit einbezieht. Ein waches Spürbewusstsein für den eigenen Organismus und den der Mitmenschen ermöglicht eine Präsenzfähigkeit, welche die intuitive Wahrnehmung öffnet und damit ein Mehr des Momentes erfassbar macht.

Im multidimensionalen Blick der Orgodynamik unterscheiden wir zwischen mentaler, emotionaler, körperlicher, energetischer, essenzieller und der Welt-Präsenz, durch die wir uns flüssig bewegen können, neugierig, ausdauernd, mal fokussiert, mal gelassen oder in entspannter Sammlung (Plesse-St. Clair 2011). Jeder Zugang ist gleichwertig darin, den Moment unmittelbar zu erfassen.

Wachsein in allen Dimensionen kann zu einer bedingungslosen Gegenwärtigkeit führen, die nicht an einem spezifisch zu erreichenden Zustand festhält. Darin wird ein umfassendes sinnliches Anwesendsein genauso kostbar, wie eine nonduale Raumerfahrung.

Präsenz, oder besser Präsentsein ist somit kein statischer Zustand, sondern ein sich ständig veränderndes Kontinuum. Im orgodynamischen Kontext geht es dabei erst einmal um einen möglichst inhalts- oder, wie man auch sagen könnte, kontextfreien Modus. Damit wird Präsenz zu einem neutralen Bewusstseinsmodus, der mit verschiedenen Inhalten gefüllt werden kann.

In multidimensionaler Präsenz, die freigeschält ist von Zielen, Kontexten, Konzepten und Vorstellungen partizipieren wir am Jetztmoment, als Teilnehmende und Gestaltende des Lebensstromes, mit all unseren Schätzen des Menschseins und den Geschenken der essenziellen Qualitäten, die da heraus entstehen wie z.B. Weite, Freundlichkeit, Schönheit, Staunen, Offenheit, Fülle, Mut, Lebendigkeit, Authentizität u.a.

Natürliche Präsenzqualitäten sind vergleichbar mit denen, die in der Liebe entstehen, sagt John Welwood, wie

„das Gefühl, Teil von etwas Größerem zu sein; ein intensives Gefühl, ich selbst zu sein, der Mensch, der ich wirklich bin; ... eine fließende Verbundenheit; ... mehr körperliche und sinnliche Lebendigkeit; ... nach Hause kommen ..." (Welwood 2002).

Präsenz ist somit der Bewusstseinsmodus, der uns Menschen nicht nur Bewusstseinsweite eröffnet, sondern uns auch mit den Qualitäten unseres tiefsten Menschsein verbindet und damit der wesentliche Schlüssel für ein humanes und bewusstes Lernen und Leben ist.

\section{Vom Flow zum Fließenden Gewahrsein (FlieG)}

Der englische Begriff Flow meint in seiner ursprünglichen Bedeutung Fließen, Strömen. Wir bezeichnen damit das Prinzip des Fließens als eine Grundqualität unserer Lebensenergie, die sich in Bewegung und Strömen ausdrückt und zwar auf allen Ebenen unseres Menschseins.

Flow kennzeichnet auch die Erfahrung, mit etwas in tiefem Einklang zu sein, wenn sich z.B. die Gleichschaltung von äußeren Ereignissen und innerer Befindlichkeit in einem anstrengungslosen Tun äußert. Der Tanz, der in flüssigen, nicht gebremsten Bewegungen geschieht, Schönheit und Anmut zum Vorschein bringt und - metaphorisch gesprochen - „den Tänzer zum Tanz werden lässt“, kann als Flow bezeichnet werden. 
Oberflächlich verwendet kann der Begriff Flow (z.B. in einem Satz wie: „Heute war ich gut im Flow“) auch eine Aussage darüber sein, dass ,alles im Fluss war“ und nach den eigenen Vorstellungen „geklappt“ hat, was ein Tiefenverständnis von Flow verhindert. Zudem wird Flow auch mit der Glückserfahrung gleichgesetzt.

Der Begriff „Flow“ wurde durch Mihaly Csikszentmihalyi bekannt. Die Erfahrung von Flow wird mit der Erfahrung von Glück gekoppelt, was durch die Kontrolle des eigenen Innenlebens erreicht werden kann (Csikszentmihalyi 1993). Er sagt, dass die optimale Erfahrung von der Fähigkeit abhängt, zu steuern, was sich in jedem Moment im Bewusstsein abspielt. Sein Ansatz ist, dass Flow das Prinzip sei, das den Menschen glücklich macht und Wohlgefühl hervorruft, wobei ein optimaler Zustand erreicht werden kann.

„Was den Menschen wirklich befriedigt, ist, sich im eigenen Leben wohl zu fühlen.“

Die optimale Erfahrung entsteht dann, wenn die Aufmerksamkeit auf ein Ziel gelenkt ist und der Mensch dadurch zeitweilig alles andere vergessen kann. Seine Untersuchungen zeigen, wie durch die Koordination von Bewusstseinsenergie eine Synchronisierung der Handlungsimpulse mit äußeren Gegebenheiten passieren kann, die wiederum die Glückserfahrung erzeugt.

Der Ansatz von Csikszentmihalyi stellt in unserem Verständnis das Ursache-Wirkungs-Prinzip zu sehr in den Vordergrund. Es entsteht ein übergeordnetes Ziel:

\section{Flow führt zu Glück und Effektivität.}

Obwohl Csikszentmihalyi an einigen Stellen deutlich darauf hinweist, dass es nicht darum geht, der Natur unsere Wünsche aufzuzwingen und die Grenzen des menschlichen Willens anzuerkennen, trägt er dazu bei, Flow auf ein Prinzip der Machbarkeit $z u$ reduzieren, wenn er $z$.B. sagt,

„... dass man sich, statt sich von anonymen Kräften herumgestoßen zu fühlen, als Herr des eigenen Schicksals fühlt"

und wenn er von der optimalen Erfahrung spricht, die etwas ist, was wir in dem freiwilligen Bemühen, etwas Schwieriges und etwas Wertvolles zu erreichen, ,herbeiführen" können.

Seine Parameter sind:

- Ziele setzten,

- sich in die Handlung vertiefen,

- Aufmerksamkeit auf das Geschehen richten,

- Lernen, sich an der unmittelbaren Erfahrung zu freuen.

Dies sind sicher sinnvolle Orientierungspunkte. Doch in unserem Verständnis wird das Glücksversprechen und die immanente Zielorientierung zum Hindernis für die authentische Erfahrung.

Eines unserer Paradigmen, das dem Verständnis von Flow am nächsten kommt, ist das Fließende Gewahrsein, FlieG.

Hierbei geht es um eine „Präsenzfähigkeit in Bewegung“, die das Kontinuum der sich aneinanderreihenden Momente im Auge behält, ohne ein festgelegtes Ziel in 
Aussicht zu stellen und die Erfahrung, welcher Art sie auch immer sein mag, als Bewusstseinsgabe anzunehmen.

\section{Dazu sind 3 Aspekte wichtig.}

- Die Bereitschaft, sich auf das Unbekannte, das Nichtwissen einzulassen, welches immer einen ungewissen Ausgang hat (natürlich kann auch das als ein Ziel verstanden werden, es lässt jedoch den Raum für die Art der Erfahrung völlig offen).

- Die Bereitschaft, die Kostbarkeit des Lebens zu würdigen, welches sich in unendlich vielen Formen hervorbringt. Dazu gehören Glückserfahrungen genauso, wie Getrenntheitserfahrungen, Einheitserfahrungen, Stagnation, Harmonie, Nichtharmonie. Die geistige Haltung, die Kostbarkeit des Lebens wahrzunehmen, lässt uns die unendliche Vielfalt bestaunen.

- Die Kunst des „Im Fluss und am Fluss Seins“ zu praktizieren.

Dazu gehört zunächst die Bereitschaft, einer Bewegung, einer Handlung, einem Geschehen die volle Aufmerksamkeit zu geben. Dabei handelt es sich jedoch nicht um eine fokussierte Präsenz allein, sondern um ein Gewahrsein, das 2 Bewusstseinsqualitäten verbindet. Wir nennen dies metaphorisch gesprochen „im Fluss der Bewegung, des Lebensstroms der Handlung und gleichzeitig am Fluss zu sein“.

Wir sind gleichzeitig - im Fluss der Erfahrung und tauchen ein mit allen Sinnen und allen Aspekten unseres Menschseins UND gleichzeitig stehen wir wie am Fluss und betrachten das Geschehen und Erleben mit einem nicht bewertenden weichen AHA. Ich gebe mich dem Geschehen hin und beachte es, während es geschieht. Zwei Bewusstseinsqualitäten kommen zusammen.

Z.B. erlaube ich die nicht zielgerichtete Bewegung einer Hand und ich bemerke diese gleichzeitig, Moment für Moment.

Von der Holographie wissen wir, dass dann, wenn 2 Lichtstrahlen in einem passenden Winkel zusammenkommen, das völlig Neue, das Dritte, hier das dreidimensionale Bild entsteht.

So kann es auch sein, dass im Prozess des FlieG, des Im-Fluss und gleichzeitig AmFluss-Seins, eine neue Bewusstseinsdimension entsteht, in der die Trennung von Bewegung und dem, der die Bewegung beobachtet, aufgelöst wird und die Wahrnehmung „es bewegt sich“ entsteht. Und mit Bewegung meinen wir nicht nur die körperliche Bewegung, sondern genauso die Bewegung des Geistes, der Gefühle, der energetisch-subtilen Ebene usw.

FlieG ist eine Bewusstseinshaltung, die alle Ebenen unseres Menschseins berühren kann. Es ist ein Gewahrsein, das alles, was sich ereignet, einbezieht und im Kontinuum eines fließenden Präsentbleibens begleitet.

\section{Übungen zur Praxis des Fließenden Gewahrseins}

Die Pause für den einen Atemzug

Das Innehalten durch Meditationen und kontemplative Methoden, in dem ein Bewusstsein für die Lücke zwischen z.B. 2 Gedanken, 2 Impulsen etc. wahrgenommen 
wird, ist in vielen Traditionen zu finden. Diese Übung hier lässt sich gut mit der Methapher eines „Zuges“, der für unseren Gedankenstrom steht, verbinden. Unsere Gewohnheit ist es, auf den Zug unserer Gedanken aufzuspringen und mit ihm in die Geschichte der Gedankenketten, in die Zukunft, in die Vergangenheit, in die „IchStory“ oder die „Du-Story“ zu „fahren“. Durch den einen Atemzug extra, der uns Zeit lässt, noch nicht zu reagieren, können wir am Bahngleis, um in dem Bild zu sprechen, stehen bleiben. Da dies ein körperlicher Vorgang ist, unterbricht er die reaktive Handlungsbereitschaft, beruhigt das Nervensystem, führt zu einer augenblicksbezogenen Körperwahrnehmung und damit zurück in die Wahrnehmung des Jetzt.

Etabliert sich eine solche „Technik“ jedoch zu einer unbewussten Gewohnheit, kann sie zu einer neuen Falle werden, welche den authentischen Fluss unserer Lebensimpulse unterbricht.

\section{Das Aha und der Aha-Flow}

In dieser Praxis geht es darum, dem, was immer im Bewusstsein auftaucht, mit einem weichen, nicht bewertenden „Aha“ zu begegnen; mit anderen Worten, bevor der nächste Gedanke, Impuls etc. auftaucht, eine kleine Lücke, eine kleine Unterbrechung herzustellen und das in einer offenen, gewährenden Haltung, die das Wahrgenommene behutsam bestaunt.

Diese „Hosentaschenübung“, das „AHA“ haben wir immer dabei, sie gibt uns die Zeit durch die kleine Pause, den nächsten authentischen Impuls wahrzunehmen und nicht in reaktiven Mustern zu bleiben. Häufig ermöglicht die kleine Lücke das Entdecken von Überraschungen und damit entsteht Platz für Heiterkeit. Annahmen werden sichtbar, ungeliebte Gefühle bekommen Platz, automatische Beziehungsmuster werden unterbrochen, Teilaspekte können fühlend wahrgenommen werden und vieles mehr. Dieses Instrument ist auch sehr hilfreich in der prozessorientierten therapeutischen Begleitung.

\section{Wie eine Beziehungssituation am Morgen aussehen könnte}

ER sagt: Du, ich möchte gerne joggen gehen.

SIE: Schon wieder zieht er sein Ding durch, ohne mich einzubeziehen. Eigentlich ist das immer so, er ist nie da für mich, ich glaub, er liebt mich nicht, na ja, dann können wir uns ja gleich trennen usw.

\section{Gleiche Ausgangssituation, mit Praxis des AHA:}

ER sagt: Du, ich möchte gerne joggen gehen.

SIE: Er möchte joggen gehen - AHA. Schon wieder zieht er sein Ding durch, ohne mich einzubeziehen - AHA. Hm, ich könnte ja mal fragen, ob das stimmt oder ob er mich dabeihaben möchte - AHA. Aber eigentlich könnte er ja auch sagen, ob er dann noch gemeinsam frühstücken will - AHA. Er steht ja immer noch in der Tür und guckt mich an - AHA. Eigentlich guckt er ganz freundlich - AHA. Vielleicht können wir ja danach was zusammen unternehmen - AHA.

Verschiedenste Antworten werden durch die Lücke, durch das Nicht-Sofort-Reagieren, möglich und das kreative Potenzial für gemeinsame Lösungen steht zur Verfügung. 
Die Heiterkeit, die entstehen kann, wenn man sich bei voreiligen Rückschlüssen ertappt, ist entspannend, bringt Gelassenheit und vielleicht ein Lächeln mehr in unser oft so schnelles Leben.

\section{Die Garuda-Meditation (früher Mahamudra-Meditation genannt)}

Diese Praxis setzt direkt an der Übung des FlieG an, die beiden Bewusstseinsströme „Im Fluss - Am Fluss“ spürend, fühlend, beobachtend zu verkoppeln.

Im Bild des Garudavogels (der im Ei schon vollständig ist - er braucht bloß die Schalen abzulegen) geht es darum, sich den innersten authentischen Impulsen zu überlassen, sie frei zu schälen von Vorstellungen, Konzepten und sich der unmittelbaren Wahrnehmung des Momentes hinzugeben. Dazu gehört die Bereitschaft im Nichtwissen zu verweilen und die Bereitschaft, die Kostbarkeit des Lebens wertzuschätzen.

In dieser Meditation, die aus 3 Phasen besteht (Einstimmung, Latihan, Gebet) ist die Körperbewegung das Medium, FlieG zu erforschen.

\section{Einstimmung}

Je nach Gruppenkontext können verschiedene mentale Orientierungen zu Anfang hilfreich sein:

„Lerne deine innersten authentischen Impulse kennen, die Du noch nicht wissen musst. Stelle deine Verbindung mit dem ,Größeren' her. Wer bist Du noch? Wer bist du, wenn du dich ganz deinem Lebensstrom überlässt?“ usw.

Konkret geht es darum, sich weich und langsam dem Fluss der Bewegungen des Körpers, dem Körperflow zu überlassen und spontanen Ausdrucksimpulsen ungehindert Raum zu geben. Dadurch wird erfahrbar, was die eigenen, authentischen Impulse in diesem Moment sind. Die Teilnehmenden lernen zu unterscheiden zwischen willentlicher Steuerung und dem rezeptiv wachen Anteilnehmen an dem, was in Bewegung geschieht.

Der Begriff „Latihan“, der der indonesischen Subudschule entliehen ist („Wirkweise des Größeren“ oder auch „Übung“) wird kurz erklärt, ohne sich zu eng auf den traditionellen Kontext zu beziehen (Plesse-St. Clair 2011).

Während nun die Körperbewegungen geschehen, werden sie mit dem weichen nichtbewertenden AHA begleitet. Atemwahrnehmung, kontemplative Einstimmung oder auch der Monolog des Leermachens

„stell dir vor, alle Gedanken die auftauchen sind Kleidungsstücke oder Dinge, die du nicht mehr brauchst. Leg sie (evtl. mit einer Geste verbunden) zur Seite ..."

unterstützen eine offene mentale Haltung und die Verlangsamung.

Es geht darum, eine offene, wache Geisteshaltung zu erzeugen, die alles, was im Geist auftaucht, erst einmal da sein lässt, dem Inhalt jedoch keine weitere Aufmerksamkeit schenkt. Eine gute Einstimmung darauf ist auch, wenn sich der/die Leiter/-in selbst vor der Cruppe „outet“ und Gedanken, Gefühle, Wahrnehmungen etc. sichtbar macht, indem vor der Demonstration alles was auftaucht laut ausgesprochen wird. 


\section{Latihan}

Das Prinzip des „Im-Fluss- und Am-Fluss-Seins“, während Bewegungen im Körper geschehen, wird erklärt und demonstriert.

\section{Eine kurze Hinführung im Wortlaut}

„Richte deine Aufmerksamkeit auf eine Hand, gib ihr mit der anderen eine kleinen Stubs, wie als würdest du einen Ball anstoßen und Du weißt nicht, wo dieser hinrollt. Die Bewegung geht kontinuierlich weiter, egal wo sie hingeht, und du brauchst es auch nicht zu wissen. Du folgst der Bewegung mit einem weichen Bemerken, einem weichen Aha, während sie weiter geschieht und nach und nach deinen ganzen Körper mit einbezieht. Du folgst der Bewegung und lässt dich mitnehmen, so als wärst du im Fluss der Bewegung und gleichzeitig am Ufer des Flusses im weichen Betrachten dessen, was geschieht. Überlass dich dem, was auftaucht, ohne es verändern zu wollen, wie ein Hingeben von Moment zu Moment. Alles, was auftaucht, ist okay - Gedanken, Bilder, Gefühle, Empfindungen - du bleibst einfach weiter dabei im Fluss und am Fluss - AHA - und dann lass auch irgendwann das innere Benennen los, keine Worte mehr, einfach ein Fließen in den Moment, von Moment zu Moment."

Es ist hilfreich, die „Bewegung in Verlangsamung“ einzuladen, wodurch der Shift zum weichen AHA leichter gelingt und Körper-Gefühls-Gedanken-Muster und Bewegungsgewohnheiten nicht so leicht greifen. Es geht nicht darum, etwas zu erreichen, sondern eher „gute Bedingungen“ für das Üben zu schaffen, sich wach durch die unterschiedlichen Erfahrungen zu bewegen, sich dem zu überlassen was ist.

Schnelle langsame Bewegungen, unterschiedliche spontane Gesten, verschiedenste Gefühlswelten, Gedanken, Einsichten, das Eintauchen in die subtile Energieebene, Ankommen in der Stille, usw.

FlieG wird damit zu einem Bewusstseinsinstrument, um in der natürlichen Lebensbewegung zu navigieren, zu surfen, aktiv-rezeptiv mitzufließen und über das Leben in weiter Offenheit zu staunen, sich als Teil von etwas Crößerem zu fühlen, ohne das benennen zu müssen und sich, wenn es sein soll, darin aufgehoben und eingebettet zu fühlen.

\section{Gebet}

Gebet im Bild des „Empfangens und Zurückgebens“ drückt sich in der „Urgeste der Verneigung“ aus. Die Arme zu heben, rezeptiv zu sein für das, was in dieser Hinwendung zum „Universum, zum Größeren“ zu uns kommt und dies in der Verneigung zur Erde zurück zu bringen, wenn die Stirn den Boden berührt (diese Geste wird mehrmals ausgeführt).

Diese universelle Geste braucht keinen spezifischen religiösen Kontext und die Teilnehmenden können dies mit ihren eigenen Referenzpunkten verbinden. Ich verneige mich vor der Erde, der Natur, Gott, Buddha, Mohamed, dem Größeren usw.

Darin kann erinnert werden, dass das Individuelle immer aufgehoben ist in etwas Crößerem und essenzielle Qualitäten, wie Dankbarkeit, Verbundenheit können entstehen.

Der Kontext der Garudameditation ist in das Verständnis transpersonaler Rituale eingebettet. 
„Jedes Ritual stellt den Bezug zu einer neuen Situation her“(Paulig 1992).

So eignet sich das Ritual, das mit unterschiedlichen Zugängen arbeitet, besonders für die Praxis des FlieG, so z.B. als Gesangsritual, Malflow, Schreibflow, Fallingritual, Wunschflow usw. aus der orgodynamischen Praxis.

\section{Form-Raum-Übung}

Hier wird in einer verlangsamten meditativen Körperbewegung bewusst zwischen Form (sanfte Anspannung der zusammenkommenden Hände vor der Brust) und dem Öffnen in den Raum (Hände und Arme bewegen sich frei) gewechselt. Die Wahrnehmung shiftet zwischen Form und Raum. In einem nächsten Schritt finden die Teilnehmenden ihren ganz eigenen fließenden Rhythmus.

\section{Im Fließenden Gewahrsein mit Fragen}

Fragen, bei denen es nicht um die schnelle Antwort geht, werden zu Sonden, die das Bewusstseinsfeld stimulieren, vielfältige Perspektiven zur Verfügung zu stellen.

Die Haltung von FlieG, sich selbst von den auftauchenden Bewusstseinsinhalten überraschen zu lassen und die Antworten als fließende Inhalte unseres Bewusstseins durchzulassen, wird durch das weiche nicht wertende AHA und durch die Bereitschaft, mit der Frage präsent zu bleiben unterstützt.

Fragen, mit denen wir in der Orgodynamik forschen, sind z.B.:

n „Sag mir, was willst Du wirklich?“

- „Und sag mir noch, was willst Du wirklich?“ oder

- „Sag mir, wie vermeidest du Beziehung?“

• „Und wie vermeidest du Beziehung noch?“" oder

- „Sag mir, wer bist Du?“

Wenn eine Frage jeweils über einen längeren Zeitraum (ca. 2o Minuten) immer wieder erneut von einem Gegenüber gestellt wird, ohne sie zu kommentieren und die Antworten einfach ausgesprochen werden, entsteht ein Abschälprozess von gewohnten Überzeugungen, Annahmen und Konzepten, welche die Weite des Bewusstseins begrenzen. Es geht darum, wach in dem Fragen präsent zu bleiben, ganz im Geiste von Rilke der sagt

„und ich möchte Sie ... bitten Geduld zu haben gegen alles Ungelöste in Ihrem Herzen und ... die Fragen selbst lieb zu haben.... leben Siejetzt die Fragen. Vielleicht leben Sie dann allmählich, ohne es zu merken, eines fernen Tages in die Antwort hinein. " (Rilke in Schönherz und Fleer 2004)

\section{Der Spiraldialog}

Hier geht es darum, eine Präsenzqualität zu praktizieren, die „bei sich sein UND beim anderen sein " praktisch erfahrbar macht und sich darin dem Bewusstseinsflow des kollektiven Feldes (mindestens 4 Personen) zu überlassen.

Jeder Teilnehmende spricht frei innerhalb einer vorgegebenen Zeitstruktur und gibt das Wort dann an den nächsten Partner im Kreis weiter. Während gesprochen wird, hören die anderen Partner einfach nur zu, ohne zu unterbrechen. Diese Kreise wiederholen sich wie in einer Spirale mehrmals. Eine klassische Form (es gibt viele Va- 
riationen) ist ein Zeitrahmen mit Kreisen von je 3-5-7-5-3-1 Minuten Dauer. Es kann ein Thema, eine Frage vorgegeben sein oder aber es bleibt ein offener Entdeckungsraum. Indem es nicht um richtig, falsch, recht oder nicht rechthaben geht (David Bohm spricht von kreativer Partizipation, die häufig durch persönlichen Ehrgeiz und starre Verteidigung verhindert wird [Bohm 1998]) entsteht ein fließendes Aufmerksamkeitsfeld, dass das co-kreative Potenzial der Bewusstseinsweite weckt.

\section{Das Dimensionen-Mandala}

Die 6 Dimensionen (aus didaktischen Gründen vereinfacht)

1. Gedanken,

2. Gefühle,

3. Körper,

4. Energie,

5. Essenz und

6. Außenwelt

werden symbolisch mit Kissen ausgelegt.

Mit einer Frage oder einem Thema oder einfach der unmittelbaren Wahrnehmung der verschiedenen Dimensionen, durchwandert der Teilnehmer die Dimensionen und spricht alles aus, was dazu auftaucht.

Dabei geht es darum, allen Wahrnehmungsinhalten mit dem „nicht bewertenden weichen AHA“ Raum zu lassen. Gerade am Anfang ist es hilfreich, ein längeres Verweilen in jeder Dimension zu ermutigen (z.B. 10 min). So wird das Eintauchen, sich den Inhalten in jeder Dimension zu überlassen, mit dem sanften Fluss des Beobachtens verbunden. Auch das bewusste Mäandern zwischen den verschiedenen Dimensionen unterstützt das Fließende Gewahrsein, das an keiner Perspektive festhält. Es zeigt sich immer wieder, wie sich durch dieses durchwandern im FlieG Wahrnehmungsfixierungen lösen und dadurch eine Weitung des Bewusstseinsraum eintritt.

Wir arbeiten in der Orgodynamik auf diese Weise auch mit anderen Modellen, das Beziehungsdreieck, das Werte-Mandala usw.

\section{Zum Abschluss}

Die Dringlichkeit einer neuen Bewusstseinskultur in unserer Zeit ist unübersehbar, wird doch jede individuelle oder kollektive Werteentscheidung von unserem menschlichen Bewusstsein aus getroffen und darin zum Motor unseres Handelns.

Ein waches Innenraum-Bewusstsein unserer menschlichen Multidimensionalität, in dem alle Ebenen unseres Menschseins gleichwertig sind, bildet eine gesunde Basis, der Vielfalt des Lebens respektvoll zu begegnen in einer bewussten Beziehung zu unserer Welt.

Multidimensionale oder viel perspektivische Präsenz ist der Meisterschlüssel, FlieC eine Form, die insbesondere den sich ständig verändernden Bewusstseinsstrom im Blick behält. Aus dem breiten Spektrum der orgodynamischen Bewusstseinsarbeit liegt uns diese Praxis deshalb so am Herzen, weil sie nicht nur die exklusive Vorrausetzung einer künstlichen Übungsumgebung braucht, sondern als Grundverständnis überall im Alltag anwendbar ist. 
„Im Fluss- und Am-Fluss-Sein“, in der Tätigkeit und Wahrnehmende der Tätigkeit zu sein, geht beim Abwaschen, Einkaufen, Babywickeln, Meditieren, Lieben, in der Debatte, im Beruf, usw.

Der Bewusstseinsweg selbst, der Moment, in dem das Leben geschieht bleibt im Fokus. Tiefe nonduale, exstatische Einheits- und Glückerfahrungen werden nicht zum angesteuerten Ziel, das den Moment verstellt, sondern zu Geschenken, zur Gnade auf dem Weg des Lebens.

Bewusstseinsweitung kann in jedem Moment geschehen und uns mit seinen essenziellen Qualitäten, wie Mitfühlen, Klarheit, Verbundenheit, Frieden usw. beschenken und unser Handeln beseelen.

So werden wir zu bewussten und wachen Teilnehmenden und Gestaltenden unseres Lebens und wirken jetzt als Teil eines kokreativen Bewusstseinsfeld hinein in unsere zukünftige Welt.

\section{Literatur}

Belschner Wilfried (2006) OTSC-PA-55-v01-2006. Institut für Psychologie, Abteilung Gesundheits- \& Klinische Psychologie Oldenburg

Bennett IG (1958) Subud. Otto Reichl Remagen

Bohm David (1998) Der Dialog, das offene Gespräch am Ende der Diskussionen. Klett Kotta Stuttgart

Csikszentmihalyi Mihaly (1993) Flow. Das Geheimnis des Glücks. Klett-Cotta Stuttgart

Goldstein J, Kornfield Jack (1987/1993) Einsicht durch Meditation. Die Achtsamkeit des Herzen. Buddhistische Einsichtmeditation für westliche Menschen. O.W. Barth München

Gottwald (2006) Neurobiologische Perspektiven zur Körperpsychotherapie. In Marlock Gustl, Weiss Halko (2006) Handbuch der Körperpsychotherapie. Schattauer Stuttgart

Paulig Frithjof (1994) Struktur des Rituals als pädagogisches Prinzip. Unveröffentlichte Diplomarbeit. J.W. Goethe Universität Frankfurt a.M.

Plesse-St. Clair Gabriele (2011) Orgodynamik, Menschen multidimensional begleiten. Ein transpersonal orientierter Praxisansatz für Therapeuten, Lehrer und Berater. Klinkhardt Bad Heilbrunn

Rilke Rainer Maria. In Schönherz R und Fleer A (2004) Rilke Projekt CD1. Bis an alle Sterne. Prolog. BMG

Rogers Carl R (1973) Entwicklung der Persönlichkeit. Kösel München

Welwood John (2002) Durch Liebe reifen. Partnerschaft als spiritueller Weg. dtv München

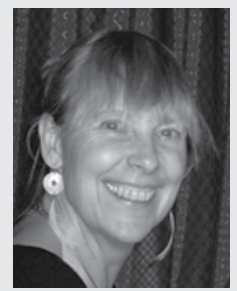

\section{Dr. phil. Gabriele Plesse-St. Clair}

Orgoville Seminare

Body-Psychotherapist (EABP), DGK, DgfE. Ausbildung u.a. Biodynamik, Unitive Psychologie, Quantumpsychologie. Begründung der Orgodynamik zusammen mit Michael Plesse (1986); Menschen multidimensional begleiten; Körper- und transpersonal-orientierte Bewusstseinsschulung. 2010 Dissertation und Buchveröffentlichung über die Orgodynamik (Klinkhardt-Verlag). 


\section{Frithjof Paulig}

Orgoville Seminare

Dipl. Päd. mit Soziologie, Psychologie.

Seit 1994 bis heute tätig als Führungskräfte- und Organisationsentwickler. Ausbildung in Orgodynamik (s.o.) und u.a. in "Geist und Leadership“ (Lassalle Inst.), Resonanz-NLP, ZEN, Ritualarbeit, Transsystemik. Seit 2009 Orgodynamik-Ausbilder. Geschäftsführer von Orgoville Seminare, Institut für Orgodynamik (DGK). 